\title{
microRNA-34a and microRNA-34c promote the activation of human hepatic stellate cells by targeting peroxisome proliferator-activated receptor $\gamma$
}

\author{
XIAOFEI LI, YONGXIN CHEN, SHUANG WU, JINKE HE, LIANQING LOU, WEIWEI YE and JINHE WANG
}

Department of Infectious Diseases, Yiwu Central Hospital, Yiwu, Zhejiang 322000, P.R. China

Received November 30, 2013; Accepted June 17, 2014

DOI: $10.3892 / \mathrm{mmr} .2014 .2846$

\begin{abstract}
Liver fibrosis is the common outcome of almost all cases of chronic liver disease. The hallmark of liver fibrosis is the activation of hepatic stellate cells (HSCs). microRNA-34a (miR-34a), which regulates a plethora of target proteins involved in the cell cycle, apoptosis, differentiation and cellular development, is found to be upregulated in both activated HSCs and liver fibrosis, while it is consistently downregulated in numerous cancer types. In the present study, the possible mechanisms underlying the role of miR-34a and miR-34c in the activation of the HSCs was investigated. Through bioinformatics analysis and a luciferase reporter assay, five genes were identified to be the target genes of miR-34a and miR-34c. Of these, peroxisome proliferator-activated receptor $\gamma(\operatorname{PPAR} \gamma)$ was selected for further investigation. Mutation luciferase reporter assay confirmed the direct interaction of PPAR $\gamma$ and miR-34a and miR-34c. Western blot analysis and quantitative polymerase chain reaction demonstrated that the expression of PPAR $\gamma$ was negatively correlated with the expression of miR-34a and miR-34c during the activation of HSCs. In activated human HSCs, inhibitors of miR-34a and miR-34c upregulated the expression of PPAR $\gamma$ and downregulated the expression of $\alpha$-smooth muscle actin. These data suggested that the miR-34 family may be involved the process of liver fibrosis by targeting PPAR $\gamma$.
\end{abstract}

\section{Introduction}

Liver fibrosis is the essential pathophysiological consequence of chronic liver injury and is characterized by the excessive accumulation of extracellular matrix (ECM) proteins, particularly collagens (1). Liver fibrosis has traditionally been regarded as an irreversible process. However, mounting clinical evidence has indicated that even advanced fibrosis is reversible (2). The

Correspondence to: Dr Yongxin Chen, Department of Infectious Diseases, Yiwu Central Hospital, 699 Jiangdong Road Yiwu, Zhejiang 322000, P.R. China

E-mail: chenyx0412@163.com

Key words: microRNA-34a, microRNA-34c, peroxisome proliferator-activated receptor $\gamma$, hepatic stellate cells, liver fibrosis activation of hepatic stellate cells (HSCs), the primary source of the ECM, that is characterized by the expression of $\alpha$-smooth muscle actin ( $\alpha$-SMA), is the pivotal process in liver fibrosis (1). Therefore, the inhibition of the accumulation of activated HSCs by modulating either their activation and/or proliferation or promoting their apoptosis is one strategy to regress liver fibrosis (1).

microRNAs (miRNAs) are a group of small, evolutionarily conserved, non-coding, naturally occurring RNA molecules, which post-transcriptionally modulate gene expression and determine cell fate by regulating multiple gene products and cellular pathways $(3,4)$. Deregulation of miRNAs has been consistently associated with a number of different human malignancies, including diseases of the liver $(5,6)$. Several miRNAs have been identified that may be involved in the process of liver fibrosis and hepatocellular carcinoma $(7,8)$.

The miR-34 family was first identified as direct transcriptional targets of p53, and is composed of miR-34a, miR34b and miR34c (9). The miR-34 family members target numerous genes, including cyclin-dependent kinases 1 and 4 , B-cell lymphoma 2, cAMP-response element binding protein (CREB), forkhead box protein P1 (Foxp1), to modulate the cell cycle, differentiation, proliferation and apoptosis (10). Lower miR-34 expression in cancer has been reported by several groups, suggesting its possible involvement in oncogenesis as a tumor suppressor (4,11-13). However, elevated miR-34 expression has been reported in activated HSCs, in rats with induced hepatic fibrosis or liver tumors and in patients with liver diseases (14-18). It has been reported that miR-34 family members may be involved in the process of liver fibrosis by targeting acyl-CoA synthetase long-chain family member 1 (ACSL1) (15). However, whether other cellular factors or proteins are involved remains elusive.

Peroxisome proliferator-activated receptor $\gamma$ (PPAR $\gamma)$ belongs to a superfamily of nuclear receptors controlling the transcriptions of numerous different genes. PPAR $\gamma$ is an important anti-fibrotic factor and is involved in the maintenance of HSCs in a quiescent phenotype (19). Several studies have reported that PPAR $\gamma$ antagonists or activators impeded the HSCs activation during live fibrosis (20-22). PPAR $\gamma$ is now considered to be a promising therapeutic target for antifibrotic chemotherapy (23).

The present study, utilizing bioinformatics and a reporter assay, investigated whether the PPAR $\gamma$ gene was a target 
protein of miR-34a/c. Expression of PPAR $\gamma$ in activated or miR-34a/c-silenced HSCs was assessed in human and rat cell models in vitro, in order to elucidate whether the miR-34 family promoted liver fibrosis by targeting PPAR $\gamma$.

\section{Materials and methods}

Cells and reagents. The immortalized human HSC line LX-2 (Institute of Cell and Molecule Biology, Central South University, Changsha, China), a generous gift from Dr. Tan (24), was maintained in Dulbecco's modified Eagle's medium (DMEM; Invitrogen Life Technologies, Carlsbad, CA, USA) supplemented with $10 \%$ fetal bovine serum (FBS; Gibco BRL, Grand Island, NY, USA), $100 \mu \mathrm{g} / \mathrm{ml}$ streptomycin (Amresco, Solon, OH, USA) and $100 \mathrm{U} / \mathrm{ml}$ penicillin (Amresco) and incubated at $37^{\circ} \mathrm{C}$ with $5 \% \mathrm{CO}_{2}$. Prior to the start of the experiments, cells were tested by PCR for mycoplasma, using commercially available primers, and were shown to be mycoplasma-negative. The cells were activated by administration of transforming growth factor $\beta 1$ (TGF- $\beta 1$; Sigma-Aldrich, St. Louis, MO, USA). Human Embryonic Kidney (HEK; Cell Center of Shanghai Institutes for Biological Sciences, Chinese Academy of Sciences, Shanghai, China) 293 cells were maintained in DMEM supplemented with $10 \%$ FBS and antibiotics at $37^{\circ} \mathrm{C}$ in $5 \% \mathrm{CO}_{2}$. The mimics and inhibitors of miR34a and miR-34c, and the respective RNA controls, were obtained from Shanghai JIMA Pharmacy Technology Co., Ltd. (Shanghai, China).

Bioinformatics approaches. To search for miRNA-34 family target genes, the online miRNA database miRanda (http://www. microrna.org), Targetscan (http://www. targetscan.org) and Pictar (http://pictar.bio.nyu.edu) were used.

Isolation and identification of rat HSCs. Primary HSCs were isolated from normal male Sprague Dawley rats aged between five and six months (weighing 400-500 g; Shanghai Laboratory Animal Center of Chinese Academy of Sciences, Shanghai, China) by in situ perfusion and density-gradient centrifugation, as previously described (25). The rats were maintained at $25^{\circ} \mathrm{C}$ on a 12/12 h light/dark cycle, with ad libitum access to rodent chow and water. The rats received humane care according to the Guide for the Care and Use of Laboratory Animals of the Chinese Academy of Sciences. The study was approved by the ethics committee of Yiwu Central Hospital. All institutional and national guidelines for the care and use of laboratory animals were followed. Isolated HSCs were suspended in DMEM supplemented with $10 \%$ FBS, and penicillin and streptomycin at a cell density of $5 \times 10^{5}$ cells $/ \mathrm{ml}$, seeded in culture flasks and cultured at $37^{\circ} \mathrm{C}$ in $5 \% \mathrm{CO}_{2}$.

Indirect immunofluorescence assay (IFA). The cells seeded on glass coverslips or 96-well plates were washed with phosphate-buffered saline (PBS) and fixed with 4\% paraformaldehyde for $20 \mathrm{~min}$ at room temperature (RT). After being washed three times with PBS, the cells were incubated in blocking buffer [PBS containing 3\% bovine serum albumin (BSA), 0.3\% Triton $^{\mathrm{TM}}$ X-100 and 10\% FBS] for at least $30 \mathrm{~min}$ and then in binding buffer (PBS containing 3\% BSA and $0.3 \%$ Triton X-100) with monoclonal antibodies (mAbs) against $\alpha$-SMA (Santa Cruz Biotechnology, Inc., Santa Cruz, CA, USA) at a dilution of 1:100 for $1 \mathrm{~h}$ at RT. Following three washes with PBS, the cells were incubated with fluorescein isothiocyanate-conjugated goat anti-mouse immunoglobulin (Ig)G (Thermo Fisher Scientific, San Jose, CA, USA) at a 1:100 dilution with binding buffer for $1 \mathrm{~h}$ at RT. The cell nuclei were stained with DAPI (Sigma-Aldrich). The stained samples were then examined with a Leica TCS SPII confocal microscope (Leica Microsystems, Wetzlar, Germany).

Total RNA extraction and quantitative polymerase chain reaction ( $q P C R)$ analysis. For general PCR, total RNA was extracted with TRIzol (Invitrogen Life Technologies) from HEK 293 cells and cDNA was synthesized using moloney murine leukemia virus reverse transcriptase (Promega Corp., Madison, WI, USA). For miRNA detection, total RNA was prepared by using the mirVana ${ }^{\mathrm{TM}}$ miRNA Isolation kit (Ambion, Austin, TX, USA) according to the manufacturer's instructions. First-strand cDNA was synthesized using the Taqman miRNA RT kit (Applied Biosystems, Foster City, CA, USA). For the detection of the miRNA levels by qPCR, TaqMan ${ }^{\circledR}$ microRNA assay (Applied Biosystems) was used to quantify the relative expression levels of miR-34a (assay ID: 000426), miR-34c (assay ID: 000428), and U6 (assay ID: 001973) as an internal control, in an Applied Biosystems 7500 Detection system (Applied Biosystems). The relative amount of miRNAs was normalized against U6 small nuclear RNA, and the fold change for each miRNA was calculated using the $2^{-\Delta \Delta C t}$ method (26). The relative miRNA expression was calculated from three different experiments.

Vector construction. The 3'-untranslated region (UTR) fragments of the indicated target mRNAs containing putative miR-34a and miR-34c binding sites were amplified by PCR from the cDNA of HEK293 cells. The amplified fragments were cloned into the $\mathrm{X} b a \mathrm{I}$ sites of pGL3-promoter vector (Promega Corp.) downstream of the luciferase coding region to generate reporter vector pGL-UTRs. The vector pGL-PPAR $\gamma$-mut with mutated binding sites was mutated with the QuikChange II XL Site-Directed Mutagenesis kit (Stratagene, La Jolla, CA, USA) according to the manufacturer's instructions. The oligonucleotides used for cloning are demonstrated in Table I. All of the primers were synthesized by Sangong Biotech Co., Ltd. (Shanghai, China). The constructed clones were confirmed by sequencing (Sangong Biotech Co., Ltd.).

Luciferase reporter assays. The HEK293 cells were plated in 24-well plates the day prior to transfection. The cells were co-transfected with internal control pRL-TK (Promega Corp.), reporter vectors and mimics of miR-34a/c or negative control probes (Shanghai JIMA Pharmacy Technology Co., Ltd.) using Lipofectamine ${ }^{\circledR} 2000$ (Invitrogen Life Technologies) according to the manufacturer's instructions. A total of $48 \mathrm{~h}$ later, the cells were harvested and applied to the luciferase measurement with a Dual-Luciferase Reporter Assay System (Promega Corp.) using a GloMax ${ }^{\circledR}$ 20/20 detection system (Promega Corp.). Values are represented as the number of relative light units (RLU).

Transient transfection. rHSCs or LX-2 cells were seeded into six- or 96-well plates the day prior to transfection. The cells 
Table I. Primers used in this study.

\begin{tabular}{ll}
\hline Primer & \multicolumn{1}{c}{ Sequence (5'-3') } \\
\hline LEF1-F & GCAGGTCTAGAGAAACATGGTGGAA \\
LEF1-R & GCAGGTCTAGACTGGGGTGCTGATG \\
Wnt2B-F & GCAGGTCTAGATGGGAAGGAGTTGTC \\
Wnt2B-R & GCAGGTCTAGAGGAGTGTTCTAGGG \\
PPAR $\gamma-\mathrm{F}$ & GCAGGTCTAGAGGACTTGTACTAGCAG \\
PPAR $\gamma-\mathrm{R}$ & GCAGGTCTAGAGGTGTCAGATTTTCC \\
PPAR $\gamma-$ mut1 & GAGTCCTGAGCCTGTCGCAACATTTCC \\
PPAR $\gamma-$ mut2 & GGAAATGTTGCGACAGGCTCAGGACTC \\
AREG-F & GCAGGTCTAGAAACAGAAAGAAGAA \\
AREG-R & GCAGGTCTAGAAATAGCATAAAAGTG \\
LDHA-F & GCAGGTCTAGACCTTGCATTTTGGGA \\
LDHA-R & GCAGGTCTAGAGGAAGAATTATGCAC \\
ACSL1-F & GCAGGTCTAGATTTCAGGTCGCAGATAG \\
ACSL1-R & GCAGGTCTAGACTGGTCCGCTTGTTG \\
FABP3-F & GCAGGTCTAGACACCACATTGCCTCATT \\
FABP3-R & GCAGGTCTAGACAAGCCTGGGTTCTGT \\
XBP1-F & GCAGGTCTAGAGGGCGCCTGCGTCGG \\
XBP1-R & GCAGGTCTAGACGGGGTGTTCTGGCC
\end{tabular}

LEF, lymphoid enhancer factor; AREG, amphiregulin; LDHA, lactate dehydrogenase; FABP, fatty acid binding protein; XBP, X box binding protein; PPAR $\gamma$, proliferator-activated receptor $\gamma$.

were transfected with inhibitors of $\mathrm{miR}-34 \mathrm{a} / \mathrm{c}$ or negative control miRNAs (Shanghai JIMA Pharmacy Technology Co., Ltd.) using Lipofectamine RNAiMAX Transfection Reagent (Invitrogen Life Technologies). Following $6 \mathrm{~h}$ of culture with the transfection mix, the cells were cultured in normal culture medium (rHSCs) or medium containing $5 \mathrm{ng} / \mathrm{ml}$ TGF- $\beta 1$ (LX-2 cells). A total of $48 \mathrm{~h}$ later, the cells were harvested and subjected to western blot analysis.

Western blot analysis. The cells were harvested in lysis buffer [50 mM Tris- $\mathrm{HCl}$ (pH 7.4), $150 \mathrm{mM} \mathrm{NaCl}, 1 \%$ Triton X-100, 1\% sodium deoxycholate, $0.1 \%$ SDS and protease inhibitor cocktail (Roche Diagnostics, Basel, Switzerland)] were first quantified by the bicinchoninic acid method (Pierce Biotechnology, Inc., Rockford, IL, USA) and then denatured by boiling for $5 \mathrm{~min}$. A total of $30 \mu \mathrm{g}$ of protein per sample were separated by $10 \%$ SDS-PAGE and transferred to a polyvinylidene difluoride membrane (Millipore, Billerica, MA, USA). The membranes were blocked with tris-buffered saline (TBS; $20 \mathrm{mM}$ Tris- $\mathrm{HCl}$ (pH 7.4), $150 \mathrm{mM} \mathrm{NaCl}$ ) containing 5\% milk for $1 \mathrm{~h}$ at $\mathrm{RT}$. Next, the membranes were incubated with monoclonal mouse antibodies against $\alpha$-SMA (1:100 dilution; sc-53142), against PPAR $\gamma$ (1:100 dilution; sc-7273) or against GAPDH (1:100 dilution; sc-365062) (all Santa Cruz Biotechnology, Inc.) for $1 \mathrm{~h}$ at $37^{\circ} \mathrm{C}$, followed by three time washes with TBS-T (TBS containing $0.1 \%$ (v/v) Tween-20) buffer. Next, the membranes were incubated with horseradish peroxidase-conjugated goat anti-mouse or anti-rabbit IgG at a dilution of 1:10,000 in TBS for $1 \mathrm{~h}$ at RT, followed by three washes with TBS-T. The proteins were then detected using the Supersignal ${ }^{\circledR}$ West Pico chemiluminescent substrate (Pierce Biotechnology, Inc.) on an AlphaEase ${ }^{\circledR}$ FC Imaging System (Alpha Innotech Corporation, San Leandro, CA, USA).

Statistical analysis. Values are presented as the mean \pm standard deviation. The software GraphPad Prism 5.0 (GraphPad Software, Inc., La Jolla, CA, USA) was used for all statistical analysis and graphical illustrations. The statistical significance was analyzed using Student t-test. $\mathrm{P}<0.01$ was considered to indicate a statistically significant difference.

\section{Results}

$P P A R \gamma$ is a target protein of $m i R-34 a$ and $m i R-34 c$. To identify the potential target mRNAs of miR-34a and miR-34c, which contain the same 'seed region', three different prediction tools (miRanda, Targetscan and Pictar) were used. Of all the predicted genes, eight genes were selected for confirmation. The criteria for target selection was not only concerning the binding possibility, but also concerning the potential functions in liver fibrosis. Therefore, the selected genes encoded proteins either correlated with certain important signaling pathways [lymphoid enhancer factor 1 (LEF1), Wnt-2B, PPAR $\gamma$, amphiregulin (AREG)] $(23,27-29)$ or associated with lipid metabolism [lactate dehydrogenase (LDHA), ACSL-1, fatty acid binding protein 3 (FABP3), X box binding protein 1 (XBP1)] $(30,31)$.

HEK293 cells were co-transfected with reporter vectors pGL-UTRs bearing the predicted binding sites and mimics of miR-34a or miR-34c together with the internal control pRL-TK. The increased expression of miR-34a or miR-34c by mimics transfection was confirmed by qPCR at $48 \mathrm{~h}$ following transfection (Fig. 1A), and a concentration of $20 \mathrm{nM}$ mimics was selected for the following experiments. The results demonstrated that the overexpression of miR-34a/34c significantly decreased the relative luciferase activity in HEK293 cells transfected with UTRs of LEF1, PPAR $\gamma$, AREG, LDHA, ACSL-1 and XBP1. LEF1, LDHA and ACSL-1 have been reported to be targeted by miR-34a/34c previously $(15,32)$. Since PPAR $\gamma$ was reported to be involved in the maintenance of a quiescent HSCs phenotype and the activation of HSCs resulted in the loss of PPAR $\gamma(19,33), \operatorname{PPAR} \gamma$ was selected for further confirmation. The reporter vector bearing the mutated UTR of PPAR $\gamma$ was constructed (Fig. 1C). It was identified that the overexpression of miR-34a/34c significantly decreased the relative luciferase activity in HEK293 cells transfected with pGL-PPAR $\gamma$ (Fig. 1D), while it caused no apparent relative luciferase activity changes in HEK293 cells transfected with pGL-PPAR $\gamma$ mut (Fig. 1E). These results indicated that PPAR $\gamma$ was a direct target gene of miR-34a and miR-34c.

Expression of miR-34a/c and PPAR $\gamma$ during the activation of HSCs. The upregulation of miR-34 family members and downregulation of PPAR $\gamma$ have been previously reported during liver fibrosis $(15,23)$. Since the activation of HSCs is the pivotal process in liver fibrosis, the expression of miR-34a/c and PPAR $\gamma$ was detected during the activation of HSCs. Rat HSCs and human HSCs (LX-2) were used in the studies.

Firstly, primary rat HSCs were isolated, and $\sim 2 \times 10^{8} \mathrm{HSCs}$ were harvested from each rat. The fraction of freshly isolated 
A

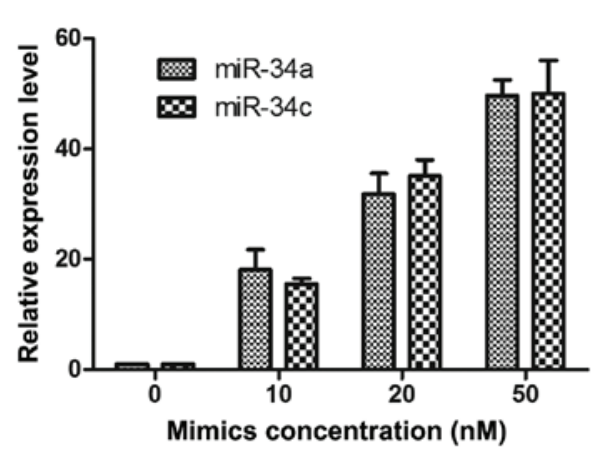

B



\section{PPARY 3'UTR 5'...CAGAGAGUCCUGAGCCACUGCCAA... Mut-PPAR.Y 3'UTR $\quad$ CUGUCGC}

D

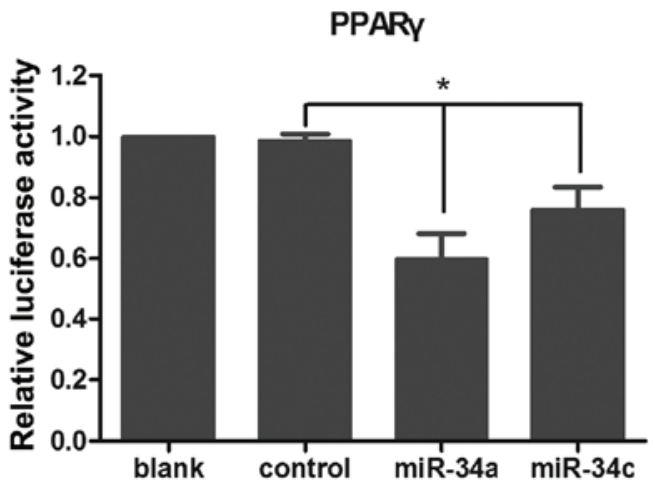

$\mathbf{E}$

mut-PPARY

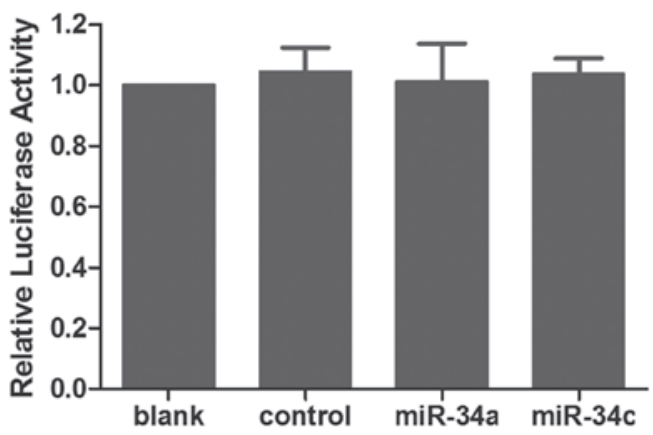

Figure 1. PPAR $\gamma$ is a direct target protein of miR-34a and miR-34c. Mimics of miR-34a and miR-34c were introduced into HEK-293 cells and upregulation of miR-34a and miR-34c was confirmed by quantitative polymerase chain reaction. (A) A concentration of $20 \mathrm{nM}$ mimics was selected for the following assays. (B) Luciferase reporter assay was used to confirm the direct interaction between miR-34a/miR-34c and target mRNAs. HEK293 cells were co-transfected with mimics, pRL-TK together with reporter vector pGL-UTRs, and the relative luciferase activity was detected. (C) The predicted miR-34a/miR-34c binding site on the PPAR $\gamma$ mRNA 3'-UTR and mutated binding site are demonstrated as bold and underlined. (D and E) Mutation luciferase reporter assay was used to further confirm the direct interaction between miR-34a/miR-34c and PPAR $\gamma$. HEK293 cells were co-transfected with mimics, pRL-TK together with (D) wild reporter vector pGL-PPAR $\gamma$ (E) and mutant reporter vector pGL-PPAR $\gamma$-mut. Relative luciferase activity was detected. Data are represented as the mean \pm standard deviation from triplicate independent experiments. ${ }^{*} \mathrm{P}<0.01$, for comparison between the miR-34a/c transfected cells and the control miRNA transfected cells. UTR, untranslated region; Mut, mutation; LEF, lymphoid enhancer factor; AREG, amphiregulin; LDHA, lactate dehydrogenase; FABP, fatty acid binding protein; XBP, X box binding protein PPAR $\gamma$, proliferator-activated receptor $\gamma$; HEK293, human embryonic kidney 293; miRNA, microRNA.

living HSCs was $\leq 90 \%$, as defined by trypan blue staining. The morphology and growth characteristics of the freshly isolated cells were observed with an inverted phase contrast microscope. The cells were small and round with the quiescent phenotype when freshly isolated; however, they demonstrated a weak adhesive growth pattern following culture for two days and presented a wall-adhesive growth pattern following culture for ten days (Fig. 2Aa-c). IFA and western blot analysis of $\alpha$-SMA were applied for demonstrating the activated phenotype of HSCs. The IFA results demonstrated that the cells expressed no or little $\alpha$-SMA on day 2 (Fig. 2Ad) and expressed abundant $\alpha$-SMA on day 10 (Fig. 2Ad). Western blot analysis also demonstrated that 
A

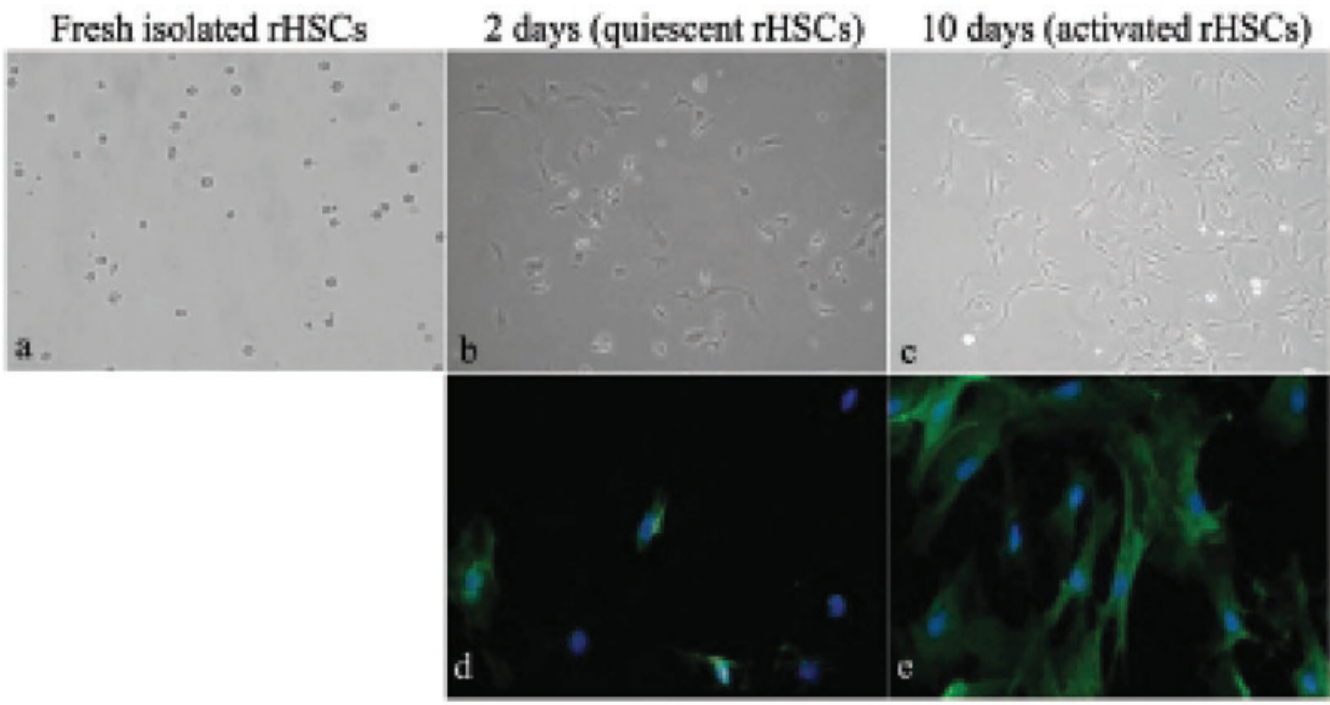

B
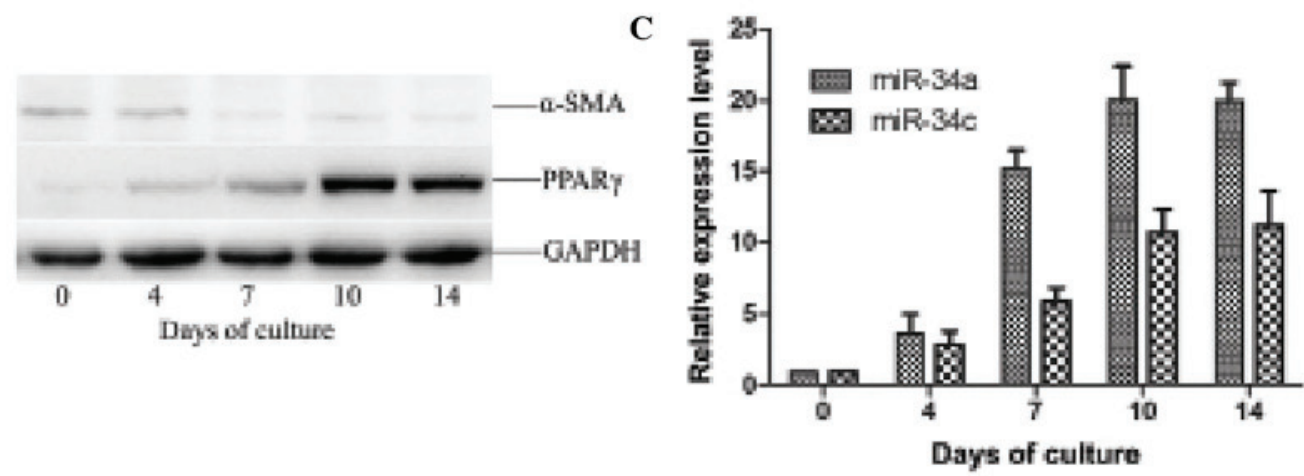

D

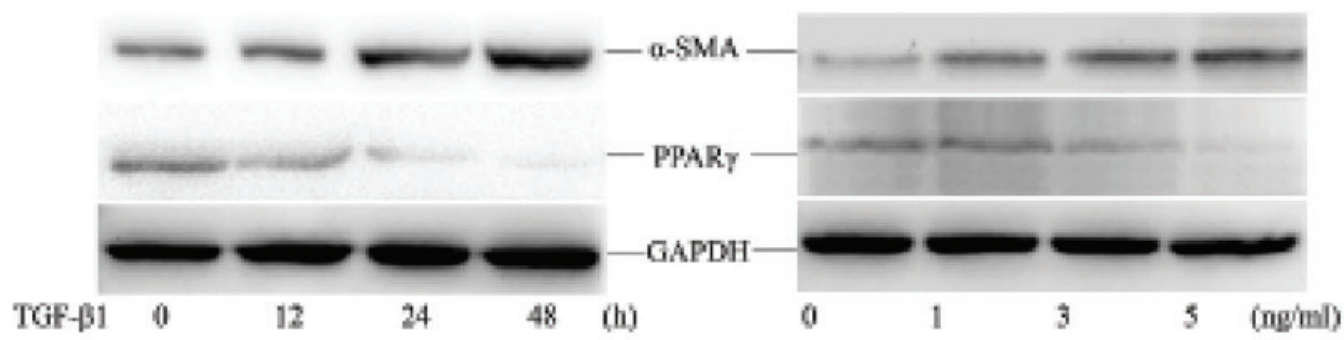

$\mathbf{E}$


Figure 2. miR-34a and miR-34c are upregulated and PPAR $\gamma$ is downregulated during the activation of HSCs. (A) Characterization of HSCs isolated from rat liver; (a) cell morphology of isolated cultured HSCs; (b and c) following isolation, the cells were cultured for (b) two days (quiescent HSCs) or for (c) 10 days (activated HSCs); (d and e) images from indirect immunofluoresence assay against $\alpha$-SMA (green) in (d) quiescent HSCs and (e) activated HSCs are demonstrated. Magnification, x100 for a-c, x400 for d-e. (B and D) Expression of $\alpha$-SMA and PPAR $\gamma$ in (B) rat HSCs and in (D) TGF- $\beta 1$-activated LX-2 cells. Cells at indicated time-points were collected and subjected to western blot analysis. GAPDH indicates the amount of protein loaded in each well. (C and E) Expression of miR-34a and miR-34c in (C) rat HSCs and (E) TGF- $\beta 1$-activated LX-2 cells. Cells at indicated time-points were collected and subjected to quantitative polymerase chain reaction. Triplicate assays were performed for each RNA sample and the relative amount of each miRNA was normalized to U6 small nuclear RNA. Data are represented as the mean \pm standard deviation. PPAR $\gamma$, proliferator-activated receptor $\gamma ;$ HSCs, hepatic stellate cells; TGF- $\beta 1$, transforming growth factor $\beta 1$; $\alpha$-SMA; $\alpha$-smooth muscle actin; HEK293, human embryonic kidney 293; miRNA, microRNA. 


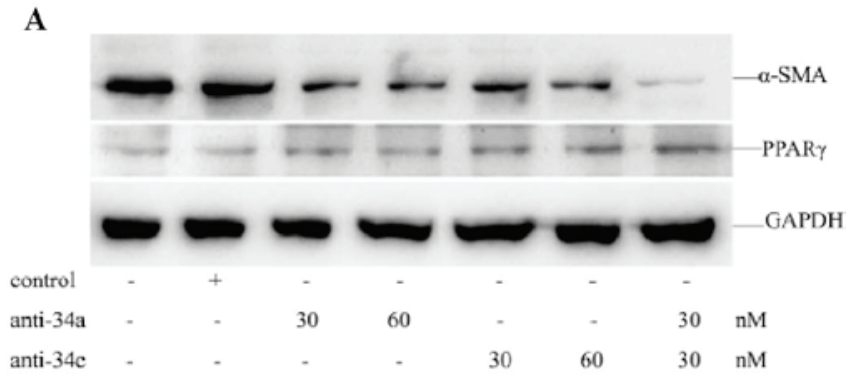

B

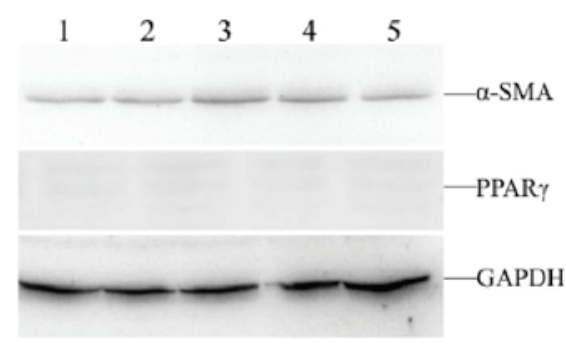

Figure 3. miR-34a and miR-34c inhibitors upregulate PPAR $\gamma$ expression and downregulate $\alpha$-SMA expression. Activated HSCs were transfected with inhibitors of miR-34a and miR-34c and the expression of $\alpha$-SMA and PPAR $\gamma$ was detected by western blot analysis. (A) TGF- $\beta 1$-activated LX-2 cells and (B) rat HSCs. Lane 1, blank; lane 2, negative control; lane 3, $100 \mathrm{nM}$ siR-34a inhibitor; lane 4, $100 \mathrm{nM}$ siR-34c inhibitor; lane 5, $50 \mathrm{nM}$ siR-34a inhibitor combined with $50 \mathrm{nM}$ siR-34c inhibitor. GAPDH indicates the amount of protein loaded in each well. PPAR $\gamma$, proliferator-activated receptor $\gamma$; HSCs, hepatic stellate cells; TGF- $\beta 1$, transforming growth factor $\beta 1 ; \alpha$-SMA; $\alpha$-smooth muscle actin; miRNA, microRNA.

the expression of $\alpha$-SMA was increased progressively with the time in culture, and reached the highest expression at day 10 (Fig. 2B). These results indicated that following culture for ten days, HSCs had been highly activated.

Next, the expression of miR-34a and miR-34c in the rat HSCs was detected by qPCR. As demonstrated in Fig. 2C, the expression of miR-34a and miR-34c in rat HSCs revealed a significant $\sim 15$-fold and 5-fold increase, respectively, following culture for seven days, and peaked with the highest expression at day 10, with an increase of $~ 20$-fold and 10-fold, respectively. The expression of miR-34a was always marginally higher than that of miR-34c.

Next, the expression of PPAR $\gamma$ was detected by western blot analysis. As demonstrated in Fig. 2B, PPAR $\gamma$ decreased progressively with the time in culture and demonstrated a negative correlation with the expression of $\mathrm{miR}-34 \mathrm{a} / \mathrm{c}$ and $\alpha$-SMA.

The expression of miR-34a/c and PPAR $\gamma$ was also analyzed during the activation of human HSC LX-2 cells. TGF- $\beta 1$ is one of the critical factors for the activation of HSC during chronic inflammation (34). LX-2 cells were treated with TGF- $\beta 1$ for the indicated time (from 0 to $48 \mathrm{~h}$ ) in $5 \mathrm{ng} / \mathrm{ml}$ or in the indicated concentration (from 0 to $5 \mathrm{ng} / \mathrm{ml}$ ) for $48 \mathrm{~h}$. The upregulated expression of $\alpha$-SMA in both conditions indicated the activation of LX- 2 by TGF- $\beta 1$ stimulation (Fig. 2D). In addition, the expression of PPAR $\gamma$, miR-34a and miR-34c demonstrated changes that were consistent with the results in the rat HSCs during the activation of LX-2 (Fig. 2D and E). In conclusion, during activation of HSCs, miR-34a and $\mathrm{miR}-34 \mathrm{c}$ were upregulated and PPAR $\gamma$ was downregulated, and the expression of PPAR $\gamma$ was negatively correlated with the expression of miR-34a and miR-34c and the degree of HSC activation.

Inhibitors of miR-34a and miR-34c upregulate the expression of PPAR $\gamma$ and downregulate the expression of $\alpha-S M A$ in human HSCs. To further investigate the association between the miR-34 family and PPAR $\gamma$ and their effect on the activation of HSCs, activated HSCs were transfected with miR-34a and miR-34c inhibitors alone or together, and the expression of PPAR $\gamma$ and $\alpha$-SMA was detected. As demonstrated in Fig. 3A, PPAR $\gamma$ was upregulated following transfection with miR34a and miR34c alone or together, compared with the negative control miRNA in TGF- $\beta 1$-stimulated LX-2 cells, while $\alpha$-SMA was downregulated in HSCs transfected with miR-34a/c compared with negative control microRNA, which suggested that activation of HSCs was reversed. Furthermore, application of the miR34a inhibitor resulted in higher expressional regulation than the miR34c inhibitor did, and the two inhibitors in combination induced the highest expressional regulation. However, in the activated rat HSCs, no expression change was observed (Fig. 3B). These results indicated that the miR-34a family accelerated the activation of human HSCs by modulating PPAR $\gamma$, and the decreased expression of PPAR $\gamma$ may have resulted from differential regulatory mechanisms in rat and human HSCs.

\section{Discussion}

The miR-34 family is transcriptionally controlled by p53 tumor suppressor protein and regulates a plethora of target proteins, which are involved in cell cycle, apoptosis, differentiation and cellular development (10). The downregulation of miR-34a has been previously identified in numerous cancer types $(12,13,35)$, and therefore, miR-34a is considered to be a tumor suppressor. However, upregulation of miR-34 has been found in numerous liver diseases from fatty liver disease to hepatocellular carcinoma (36-38). It was reported that miR-34 family members may target ACSL1, which has a central role in lipid metabolism and fatty acid metabolism in the liver, and impairs the lipid metabolism in the liver, resulting in the development of hepatic fibrosis (15). Whether other factors are involved in this process has yet to be elucidated.

In the present study, using a bioinformatics approach, eight proteins were selected for experimental confirmation. Among them, LEF1, Wnt-2B, PPAR $\gamma$ and AREG have been reported to be correlated with several signaling pathways, including Wnt and PPAR (23,27-29), which may have an important role during liver fibrosis, while LDHA, ACSL-1, FABP3 and XBP1 were reported to be associated with lipid metabolism $(30,31)$. ACSL-1 is also used as a positive control since it has been reported to be the target of miR-34a and miR-34c (15). The reporter assay indicated that LEF1, PPAR $\gamma$, AREG, LDHA, ACSL-1 and XBP1 are possible target genes of miR-34a and miR-34c. Among these, PPAR $\gamma$ has been reported to be potently involved in liver fibrosis (23). Therefore, PPAR $\gamma$ was selected for further analysis in the present study. The mutation reporter assay confirmed that PPAR $\gamma$ is the direct target of miR-34a and miR-34c. 
PPAR $\gamma$ is a ligand-activated nuclear transcription factor that belongs to the nuclear hormone receptor superfamily. PPAR $\gamma$ has a key role in HSC biology and is involved in the maintenance of a quiescent HSC phenotype (19). PPAR $\gamma$ receptors were found to have anti-proliferative and anti-fibrotic effects on activated HSCs, as well as to induce HSC apoptosis through a mechanism involving an extrinsic apoptosis pathway $(20,23)$.

It has been reported previously that miR-34 family members are upregulated and PPAR $\gamma$ is downregulated during liver fibrosis $(15,23)$. Considering the hallmark of liver fibrosis is the activation of HSCs, the present study next detected the expression of miR-34 family members and PPAR $\gamma$ during the activation of HSCs. The results demonstrated that miR-34 family member expression was increased, while PPAR $\gamma$ expression was reduced during the activation of HSCs both in rats and humans, which is consistent with previous studies $(15,23)$. Furthermore, the expression of PPAR $\gamma$ was negatively correlated with the expression of miR-34 family members and the degree of activation.

The present study further examined the association of miR-34 and PPAR $\gamma$ in activated HSCs by using miR-34 inhibitors. The results from the human HSCs demonstrated that PPAR $\gamma$ was upregulated and $\alpha$-SMA was downregulated when the cells were transfected with miR-34 and miR-34c inhibitors alone or in combination, indicating that miR-34a and miR-34c inhibitors may decrease the activation of HSCs by upregulating the expression of PPAR $\gamma$. The miR-34 family may exhibit profibrotic effects by targeting PPAR $\gamma$. However, no expression changes of PPAR $\gamma$ and $\alpha$-SMA were observed in rat HSCs transfected with miR-34 and miR-34c inhibitors alone or in combination. Further bioinformatics analysis demonstrated that the binding site of human PPAR $\gamma$ mRNA is located in a poorly conserved region in mammals, and there were no predicted binding sites for miR-34a and miR-34c on the 3'UTR of rat PPAR $\gamma$ mRNA. This is not in accordance with the general observation that binding sites are always conserved in species, while the results from the mutation reporter assay confirmed this interaction. These data suggested that although the expression of miR-34 family members, PPAR $\gamma$ and $\alpha$-SMA demonstrated a similar expression pattern in rat HSCs and in human HSCs, the regulation mechanism in the two cell lines may be contrasting. It has been previously reported that TGF- $\beta 1$ inhibits the expression of PPAR $\gamma$ in activated rat HSCs through the $\beta$-catenin pathway (39). TGF- $\beta 1$ is one important cytokine expressed following liver injury and is the most important cytokine stimulating fibrogenesis in HSCs (1). It may be possible that in rat HSCs, the inhibition of PPAR $\gamma$ is mainly the result of the upregulation of TGF- $\beta 1$ during the activation of HSCs, while in human HSCs, the inhibition of PPAR $\gamma$ results from the combination effect of upregulation of TGF- $\beta 1$ and miR-34a/c.

For the first time, to the best of our knowledge, the present study identified and confirmed PPAR $\gamma$ to be a target gene of the miR-34 family. The regulation of the miR-34 family is negatively associated with PPAR $\gamma$ in activated HSCs. These data suggested that miR-34 family members may be involved in liver fibrosis by targeting PPAR $\gamma$.

\section{References}

1. Bataller R and Brenner DA: Liver fibrosis. J Clin Invest 115: 209-218, 2005.
2. Moreira RK: Hepatic stellate cells and liver fibrosis. Arch Pathol Lab Med 131: 1728-1734, 2007.

3. Bartel DP: MicroRNAs: genomics, biogenesis, mechanism, and function. Cell 116: 281-297, 2004.

4. Wiggins JF, Ruffino L, Kelnar K, et al: Development of a lung cancer therapeutic based on the tumor suppressor microRNA-34. Cancer Res 70: 5923-5930, 2010.

5. Raisch J, Darfeuille-Michaud A and Nguyen HT: Role of microRNAs in the immune system, inflammation and cancer. World J Gastroenterol 19: 2985-2996, 2013.

6. Vettori S, Gay S and Distler O: Role of MicroRNAs in Fibrosis. Open Rheumatol J 6: 130-139, 2012.

7. Noetel A, Kwiecinski M, Elfimova N, Huang J and Odenthal M: microRNA are central players in anti- and profibrotic gene regulation during liver fibrosis. Front Physiol 3: 49, 2012.

8. Katayama Y, Maeda M, Miyaguchi K, et al: Identification of pathogenesis-related microRNAs in hepatocellular carcinoma by expression profiling. Oncol Lett 4: 817-823, 2012.

9. He L, He X, Lim LP, et al: A microRNA component of the p53 tumour suppressor network. Nature 447: 1130-1134, 2007.

10. Chen F and Hu SJ: Effect of microRNA-34a in cell cycle, differentiation, and apoptosis: a review. J Biochem Mol Toxicol 26: 79-86, 2012.

11. Trang P, Wiggins JF, Daige CL, et al: Systemic delivery of tumor suppressor microRNA mimics using a neutral lipid emulsion inhibits lung tumors in mice. Mol Ther 19: 1116-1122, 2011.

12. Pang RT, Leung CO, Lee CL, et al: MicroRNA-34a is a tumor suppressor in choriocarcinoma via regulation of Delta-like1. BMC Cancer 13: 25, 2013.

13. Yan K, Gao J, Yang T, et al: MicroRNA-34a inhibits the proliferation and metastasis of osteosarcoma cells both in vitro and in vivo. PLoS One 7: e33778, 2012.

14. Pogribny IP, Starlard-Davenport A,Tryndyak VP, et al: Difference in expression of hepatic microRNAs miR-29c, miR-34a, miR-155, and miR-200b is associated with strain-specific susceptibility to dietary nonalcoholic steatohepatitis in mice. Lab Invest 90: 1437-1446, 2010

15. Li WQ, Chen $\mathrm{C}, \mathrm{Xu}$ MD, et al: The rno-miR-34 family is upregulated and targets ACSL1 in dimethylnitrosamine-induced hepatic fibrosis in rats. FEBS J 278: 1522-1532, 2011.

16. Guo CJ, Pan Q, Li DG, Sun H and Liu BW: miR-15b and miR-16 are implicated in activation of the rat hepatic stellate cell: An essential role for apoptosis. J Hepatol 50: 766-778, 2009.

17. Murakami Y, Toyoda $\mathrm{H}$, Tanaka M, et al: The progression of liver fibrosis is related with overexpression of the miR-199 and 200 families. PLoS One 6: e16081, 2011.

18. Kota J, Chivukula RR, O'Donnell KA, et al: Therapeutic microRNA delivery suppresses tumorigenesis in a murine liver cancer model. Cell 137: 1005-1017, 2009.

19. Hazra S, Xiong S, Wang J, et al: Peroxisome proliferator-activated receptor gamma induces a phenotypic switch from activated to quiescent hepatic stellate cells. J Biol Chem 279: 11392-11401, 2004.

20. Sharvit E, Abramovitch S, Reif S and Bruck R: Amplified inhibition of stellate cell activation pathways by PPAR- $\gamma$, RAR and RXR agonists. PLoS One 8: e76541, 2013.

21. Attia YM, Elalkamy EF, Hammam OA, Mahmoud SS and El-Khatib AS: Telmisartan, an AT1 receptor blocker and a PPAR gamma activator, alleviates liver fibrosis induced experimentally by Schistosoma mansoni infection. Parasit Vectors 6: 199, 2013.

22. Bruck R, Weiss S, Aeed H, Pines M, Halpern Z and Zvibel I: Additive inhibitory effect of experimentally induced hepatic cirrhosis by agonists of peroxisome proliferator activator receptor gamma and retinoic acid receptor. Dig Dis Sci 54: 292-299, 2009.

23. Zhang F, Kong D, Lu Y and Zheng S: Peroxisome proliferator-activated receptor- $\gamma$ as a therapeutic target for hepatic fibrosis: from bench to bedside. Cell Mol Life Sci 70: 259-276, 2012.

24. Guo GH, Tan DM, Zhu PA and Liu F: Hepatitis B virus X protein promotes proliferation and upregulates TGF-beta1 and CTGF in human hepatic stellate cell line, LX-2. Hepatobiliary Pancreat Dis Int 8: 59-64, 2009.

25. Weiskirchen R and Gressner AM: Isolation and culture of hepatic stellate cells. Methods Mol Med 117: 99-113, 2005.

26. Livak KJ and Schmittgen TD: Analysis of relative gene expression data using real-time quantitative PCR and the 2(-Delta Delta C(T)) method. Methods 25: 402-408, 2001.

27. MaoCD and Byers SW: Cell-contextdependent TCF/LEFexpression and function: alternative tales of repression, de-repression and activation potentials. Crit Rev Eukaryot Gene Expr 21: 207-236, 2011. 
28. Cha YH, Kim NH, Park C, Lee I, Kim HS and Yook JI: MiRNA-34 intrinsically links p53 tumor suppressor and Wnt signaling. Cell Cycle 11: 1273-1281, 2012.

29. Busser B, Sancey L, Brambilla E, Coll JL and Hurbin A: The multiple roles of amphiregulin in human cancer. Biochim Biophys Acta 1816: 119-131, 2011.

30. Lee AH, Scapa EF, Cohen DE and Glimcher LH: Regulation of hepatic lipogenesis by the transcription factor XBP1. Science 320 1492-1496, 2008.

31. Hanhoff T, Lücke C, Spener F: Insights into binding of fatty acids by fatty acid binding proteins. Mol Cell Biochem 239: 45-54, 2002.

32. Kaller M, Liffers ST, Oeljeklaus S, et al: Genome-wide characterization of miR-34a induced changes in protein and mRNA expression by a combined pulsed SILAC and microarray analysis. Mol Cell Proteomics 10: M111.010462, 2011.

33. Marra F, Efsen E, Romanelli RG et al: Ligands of peroxisome proliferator-activated receptor gamma modulate profibrogenic and proinflammatory actions in hepatic stellate cells. Gastroenterology 119: 466-78, 2000.
34. Inagaki Y, Okazaki I: Emerging insights into transforming growth factor beta Smad signal in hepatic fibrogenesis. Gut 56: 284-292, 2007.

35. Yang S, Li Y, Gao J et al: MicroRNA-34 suppresses breast cancer invasion and metastasis by directly targeting Fra-1. Oncogene 32: 4294-4303, 2013.

36. Castro RE, Ferreira DM, Afonso MB, et al: miR-34a/SIRT1/p53 is suppressed by ursodeoxycholic acid in the rat liver and activated by disease severity in human non-alcoholic fatty liver disease. J Hepatol 58: 119-125, 2013.

37. Meng F, Glaser SS, Francis $\mathrm{H}$ et al: Epigenetic regulation of miR-34a expression in alcoholic liver injury. Am J Pathol 181: 804-817, 2012.

38. Pineau P, Volinia S, McJunkin K et al: miR-221 overexpression contributes to liver tumorigenesis. Proc Natl Acad Sci USA 107: 264-269, 2009.

39. Qian J, Niu M, Zhai X, Zhou Q and Zhou Y: $\beta$-Catenin pathway is required for TGF-betal inhibition of PPAR $\gamma$ expression in cultured hepatic stellate cells. Pharmacol Res 66: 219-225, 2012. 